\title{
6.3 Reconstruction of attractors of complex systems on the basis of time series
}

Annotation: Signs of chaotic behavior were found in the time series of technological indicators of the sugar refinery defecosaturation station systems. Studies confirm the hypothesis of nonlinear complex behavior of a dynamic control object, the presence in its behavior of deterministic chaos, the phenomena of intermittency and attractive behavior.

Keywords: time series, control system, sugar production, chaotic behavior.

The technological complex of a sugar refinery has complex behavior, including chaoticIn [318].

A time series is usually a sequence of events observed at some generally equal time intervals. In relation to the technology of purification of diffusion juice, this parameter can be the $\mathrm{pH}$ value, dry matter concentration, consumption of purified juice.

The main idea of applying the methods of chaotic dynamics to the analysis of time series is that the basic structure of a chaotic system containing all the information about the system, namely the attractor of a dynamic system (a subset of phase space that attracts trajectories in infinite time), can be restored through the measurement of only one observed characteristic of this dynamic system, fixed as a time series. According to the method of Grasberger and Procacci, the procedure of reconstruction of the phase space and restoration of the chaotic attractor of the system in the dynamic analysis of the time series is reduced to the construction of the so-called phase space.

The following software products were used for dynamic data analysis: DATAN's Dataplore program, a set of TISEAN modular programs created at the Institute of Physics and Theoretical Chemistry of the University of Frankfurt by Rainer Hegger, Holger Kantz and Thomas Schreiber, Chaos Data Analyzer (CDA), written by J. Sprott and J. Rowlands, as well as the Fractan program, developed at the Institute of Mathematical Problems of Biology of the Russian Academy of Sciences by V. Sychev. For fractal analysis, we used a specialized set of extensions of the MatLab program FracLab, created in the French laboratory Fractals team. 
The main idea of applying the methods of chaotic dynamics to the analysis of time series is as follows. It turns out [319] that the basic structure of a chaotic system, which contains all the information about the system, namely, its attractor (a subset of phase space that attracts trajectories within infinite time), can be restored by measuring only one observed parameter of this dynamic system, fixed as a time series.

Deterministic dynamical systems describe the evolution of a system over time in some phase space

$$
\Gamma \subset R^{d}
$$

These systems can be generated, for example, by ordinary differential equations

$$
\dot{x}=F(x(t))
$$

or, if time is discrete $t=n \Delta t$, an expression of the form

$$
x_{n+1}=f\left(x_{n}\right)
$$

Time series can then be considered as a sequence of observations $\left\{\mathrm{S}_{\mathrm{n}}=\mathrm{S}\left(\mathrm{x}_{\mathrm{n}}\right)\right\}$. Because the sequence (usually scalar) $\left\{S_{n}\right\}$ does not in itself generate a multidimensional phase space of a dynamic system, it is necessary to use some technique to reveal the multidimensional structure using only the available data.

According to the method of Grasberger and Procacci [319], the procedure of reconstruction of phase space and restoration of chaotic attractor of the system in dynamic time series analysis is reduced to construction of so-called lag or restored space using the method of delays. Vectors in the new space, attachment space, formed from the values of the time series of scalar measurements with time delay:

$$
\bar{S}_{n}=\left(S_{n-(m-1) r}, S_{n-(m-2) r}, \ldots, S_{n}\right)
$$


The number of elements $m$ is called the dimension of the attachment, the time $\tau$ is usually called the delay or lag. The theorems of Tuckens [320] and Sawyer [321] show that if the sequence $\left\{S_{n}\right\}$ actually consists of scalar measurements of the structure of a dynamical system, then, under certain assumptions, such a reconstruction of the phase portrait is an exact picture of the real set $\{x\}$, if, $m$ quite large. Or, in other words, the real attractor of a dynamic system and the "attractor" restored in the lag space in time series according to the above rule (pseudo-attractor), with adequate selection of the dimension of the embedding $m$, are topologically equivalent.

To calculate the characteristics of the pseudoattractor, such as its fractal dimension, Lyapunov exponents, it is necessary to have a set of points defined in the phase space of dimension $\mathrm{m}$ and belonging to the attractor. The number of points $M$ in the calculations is finite, but should be large enough. According to the formula proposed in [322]

$$
M \geq M_{\min }=10^{2+0.4 D}
$$

where $D$ is the dimension of the attractor. Based on this, we chose a number of data that would fully correspond to the small dynamics of motion, with a dimension $\mathrm{m}$ from 4 to 8 . According to these considerations, it was decided to choose the length of time series $\approx 16000$ measurements.

The traditional way to select the time delay is to calculate the autocorrelation function of the time series:

$$
A(\tau)=\frac{1}{m} \sum_{k=0}^{m-1}\left(S_{k}-\bar{S}\right)\left(S_{k+\tau}-\bar{S}\right) ; m=M-\tau
$$

The delay $\tau$ is chosen equal to the time of the first intersection of zero of the autocorrelation function. Another method requires the calculation of the power spectrum of the time series, ie the Fourier transform rate of the autocorrelation function. If there are multiple peaks in the power spectrum, then the delay $\tau$ is chosen equal to a quarter of the period of the highest of the dominant frequencies. The third 
method is based on calculating the function of delayed mutual information. The mutual information function $S$ is determined by the formula:

$$
S=-\sum_{i j} p_{i j}(\tau) i n \frac{p_{i j}(\tau)}{p_{i} p_{j}}
$$

where for some data breakdown $p_{i}$ is the probability of finding the value of the time series in the $i$-th interval of the breakdown, and $P_{i j}(\tau)$ is the total probability that the observed value will fall into the $i$-th interval, and the time of this observation will fall into the $j$-th interval .

The idea of software implementation of the method is as follows. First, the phase space is restored using the method of Grasberger and Procacci. The vectors in this space are determined according to formula (3). For each point $S_{i}$ of the time series, its nearest neighbor $S_{j}$ in $m$-dimensional space is sought. The distance between them is calculated:

$$
\left\|\overline{S_{i}}-\overline{S_{j}}\right\|
$$

Next, we integrate both points and calculate:

$$
R_{i}=\frac{\left|\bar{S}_{i+1}-\bar{S}_{j+1}\right|}{\left\|\bar{S}_{i}-\bar{S}_{j}\right\|}
$$

If $R_{i}$ exceeds this heuristic threshold $R_{t}$, then the point is considered to have an erroneous nearest neighbor.

One of the tests used in practice to verify the presence of a chaotic component in the studied series of financial data is to study the properties of the correlation sum $C_{m}(r)$ and the behavior of the correlation dimension $D_{m}(r)$ depending on the dimension of the investment $m$. The correlation sum $C_{m}(r)$ is the probability that the pairs of points on the reconstructed attractor in the $m$-dimensional lag space are within the distance $\mathrm{r}$ from each other. If the graph of the function $\ln C_{m}(r)$ with respect to $\ln r$ has 
a clearly defined linear section, this indicates the existence of a self-similar geometry of the attractor, which, in turn, indicates the chaotic nature of the process.

In the case of stylized data, when we know the dimension $n$ of the phase space of the dynamical system and all $n$ coordinates of each point on the attractor, the correlation dimension $D_{2}$ of the attractor is found as follows: we consider the correlation integral $C(r)$ showing the relative number of pairs of attractor points at a distance not greater than $r$ :

$$
C(r)=\frac{2}{m(m-1)} \sum_{i=0}^{m-2} \sum_{j-i+1}^{m-1} \theta\left(r-p\left(x_{i}, x_{j}\right)\right)
$$

where $\theta$ is the Heaviside function, $p$ is the distance in the $n$-dimensional phase space, $m$ is the number of points $x_{i}$ on the attractor. On rather small scales of lengths and when the dimension of embedding $\mathrm{m}$ is not less than the topological dimension of the attractor, the dependence is fulfilled:

$$
C(r) \rightarrow r^{D_{2}}
$$

where $D_{2}$ is the desired correlation dimension of the attractor. Prologarithmizing equation (11):

$$
\ln C(r) \rightarrow D_{2} \ln r
$$

Expression (12) gives the desired estimate of the dimension of the attractor as a tangent of the angle of inclination of the line, which approximates the graph of the correlation integral $C(r)$ on a double logarithmic scale.

The Hearst index is calculated using the Rescaled Range (R/S) Analysis method. The main purpose of calculating the Hearst index is to determine the longterm correlation in the time series, and to identify its fractal structure. In addition, we note that with the help of $R / S$-analysis it is possible to detect existing statistical cycles in the dynamics of the system [319].

In accordance with the value of the Hirst index $H$, all time series can be classified into three types [323]: 
- $\quad$ antipersistent time series $(0<H<0.5)$;

- $\quad$ random time series $(H=0.5)$;

- $\quad$ persistent time series $(0.5<H<1)$.

Studies of the values of the Hirst index were conducted in a software environment - Fractan.

It was noted above that determining the correlation dimension of the time series is one of the main tests used in practice to determine the presence of a chaotic component. Estimation of the correlation dimension should be performed for data logarithms [321]. Of all the software products used in the research, only two programs perform the correlation dimension assessment procedure: CDA and Fractan.

Based on the results obtained, we can conclude that the nature of the processes that take place at the defecosaturation station is mainly persistent $(0.5<H<1)$.

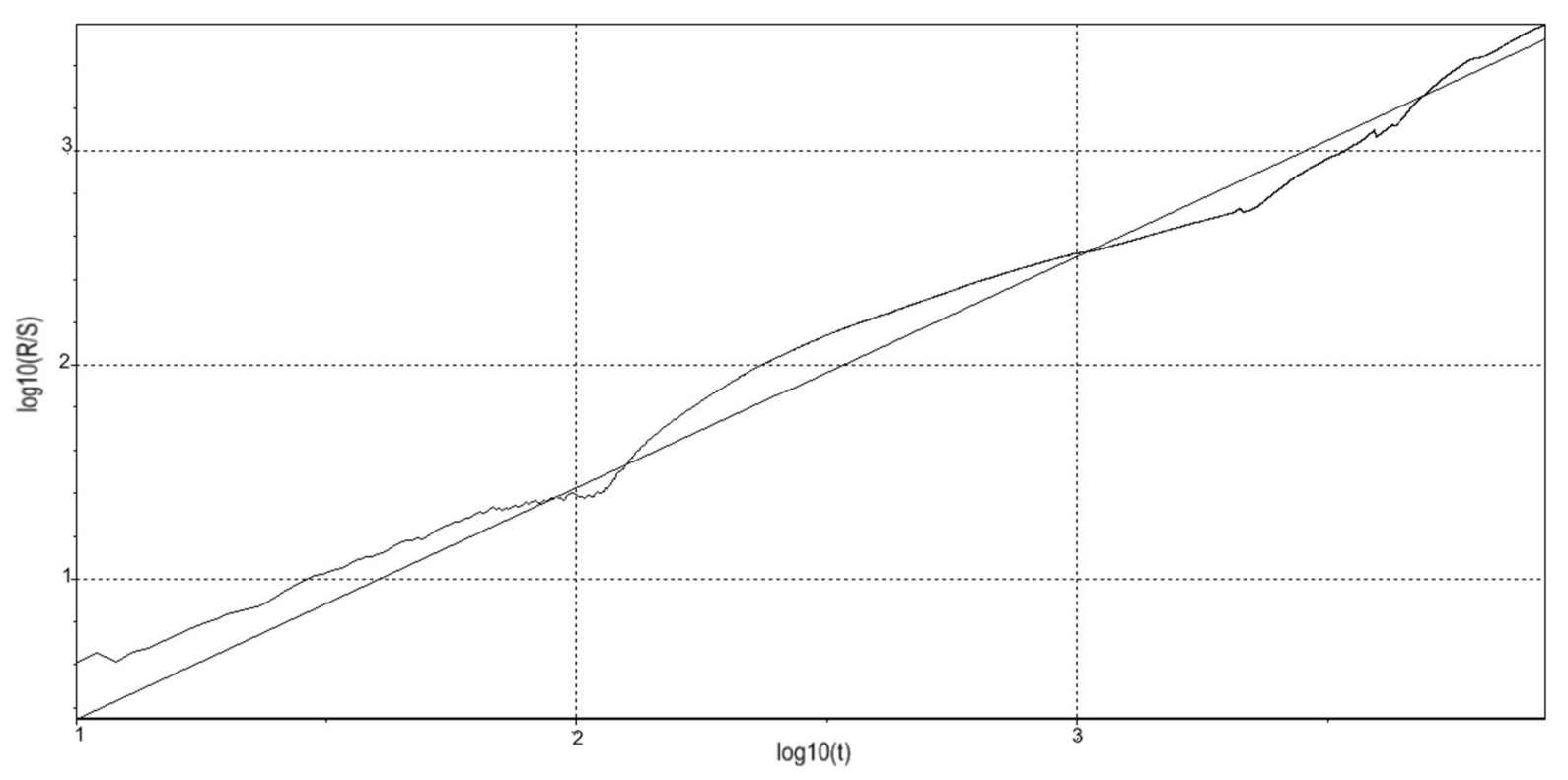

Fig. $1 . R / S$ - analysis of the studied $\mathrm{pH}$ data. 


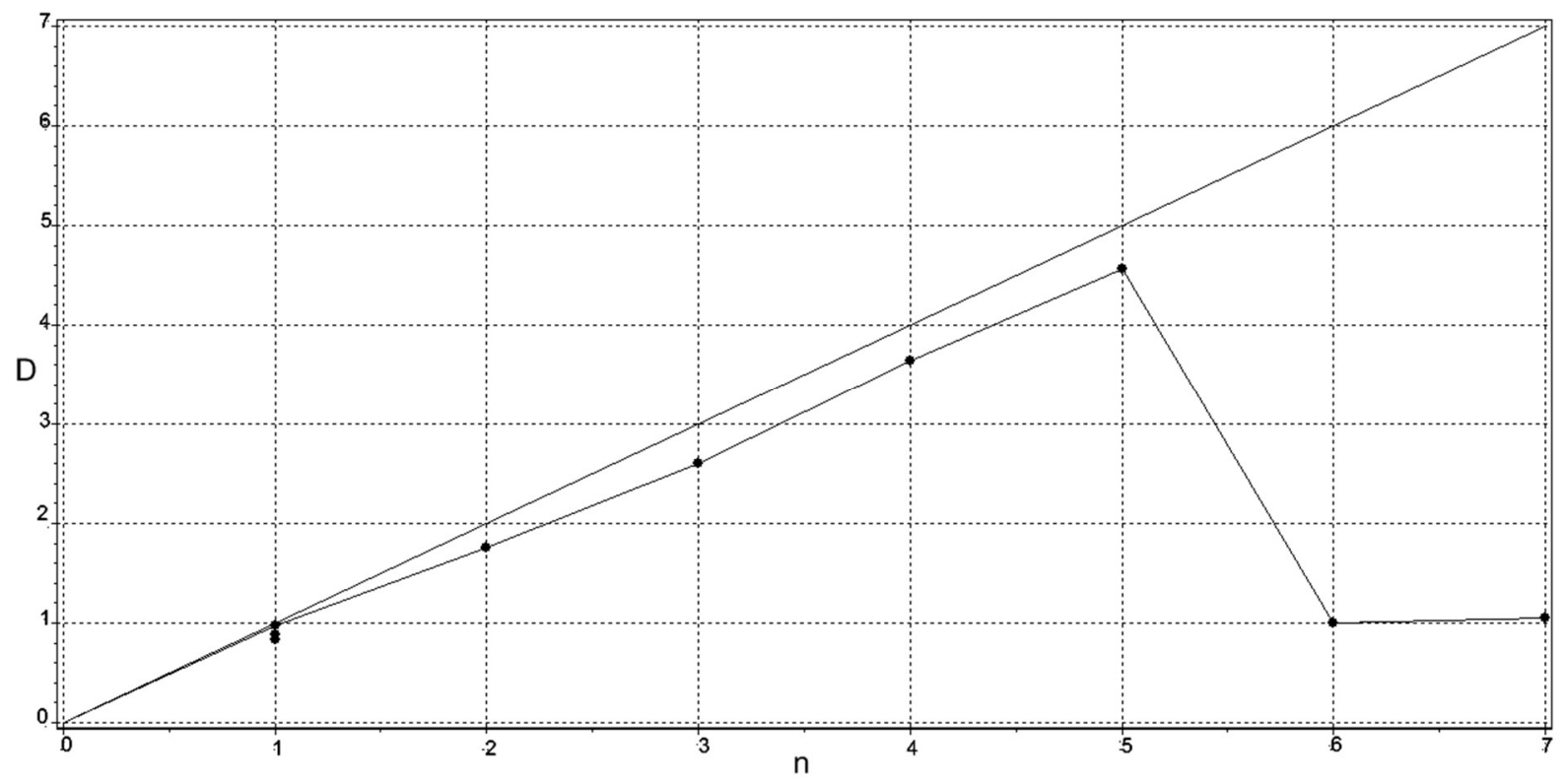

Fig. 2. Dependence of correlation dimension on dimension attachments for a number of $\mathrm{pH}$ values.

The results of the study $R / S$ - data analysis are given in table 1 .

Table 1. Hirst index, for $p H$

\begin{tabular}{|c|c|c|}
\hline Working hours & Hirst index, $\mathrm{N}$ & Fractal dimension, $D$ \\
\hline 1 & 2 & 3 \\
\hline 1 & 1.0775 & 0.9225 \\
\hline 2 & 0.7435 & 1.2565 \\
\hline 3 & 0.6403 & 1.3597 \\
\hline 4 & 1.0639 & 0.9361 \\
\hline 5 & 0.6802 & 1.3198 \\
\hline 6 & 0.6397 & 1.3603 \\
\hline 7 & 0.610 & 1.3990 \\
\hline 8 & 1.0647 & 0.9353 \\
\hline 9 & 0.8445 & 1.1555 \\
\hline 10 & 1.1641 & 0.8359 \\
\hline
\end{tabular}

The results of the study of the data of the correlation dimension and the dimension of the phase space are given in table 2 . 
Table 2. Dependence of the correlation dimension on the dimension of the attachment

\begin{tabular}{|c|c|c|}
\hline DS work time & Correlation dimension, $D_{2}$ & $\begin{array}{c}\text { The dimension of the phase } \\
\text { space, } n\end{array}$ \\
\hline 1 & 2 & 3 \\
\hline 1 & 4,563 & 5 \\
\hline 2 & 5,468 & 7 \\
\hline 3 & 3,233 & 6 \\
\hline 4 & 2,334 & 4 \\
\hline 5 & 2,474 & 7 \\
\hline 6 & 2,205 & 4 \\
\hline 7 & 2,902 & 7 \\
\hline 8 & 0.185 & 3 \\
\hline 9 & 1,437 & 7 \\
\hline 10 & 5,712 & 3 \\
\hline
\end{tabular}

As an example, below are the time series of changes in the flow of juice from defecosaturation (Fig. 3), the $\mathrm{pH}$ of the second saturation (Fig. 4) and the pressure of the saturation gas (Fig. 5).

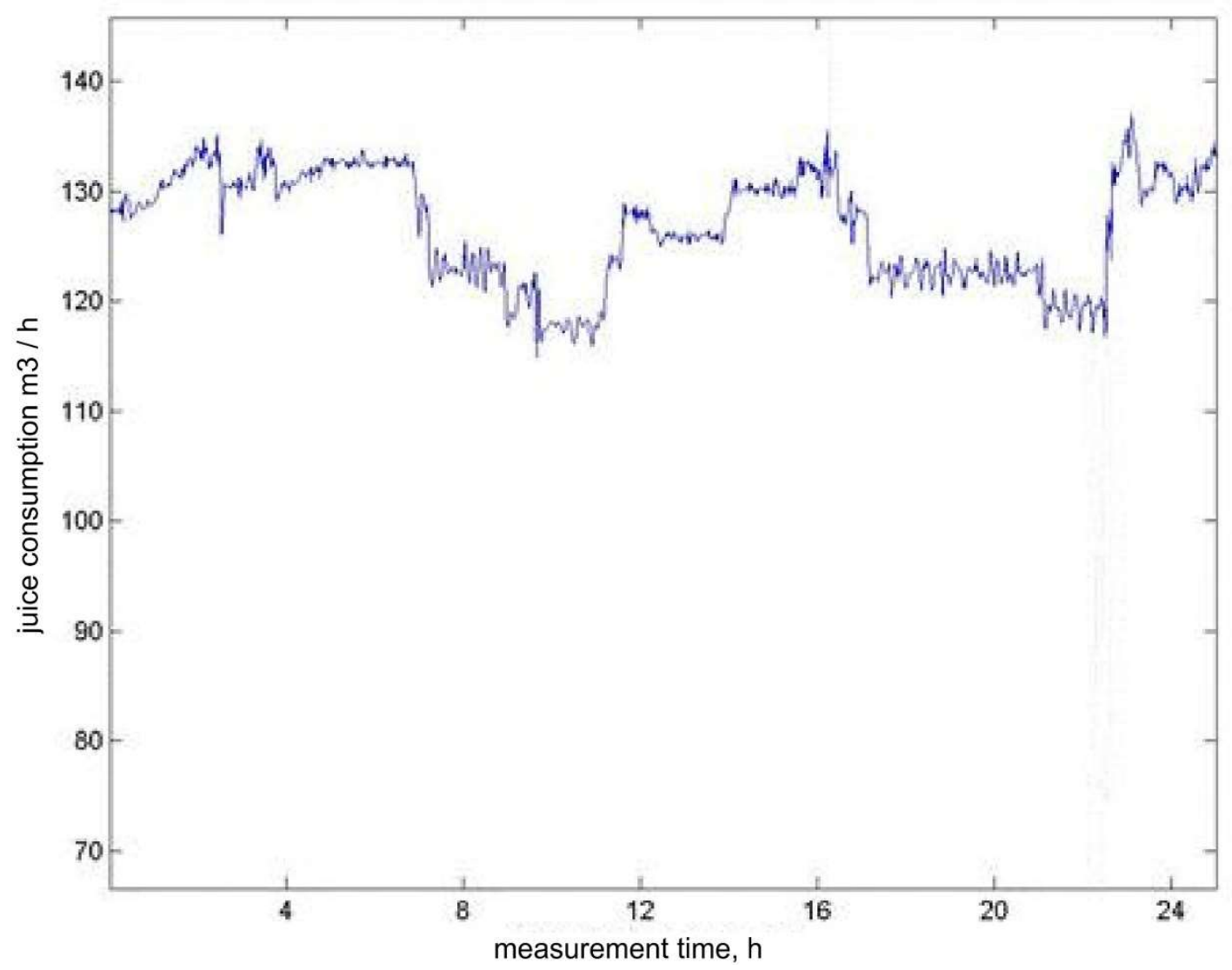

Fig. 3. Time series of juice consumption from defecosaturation. 


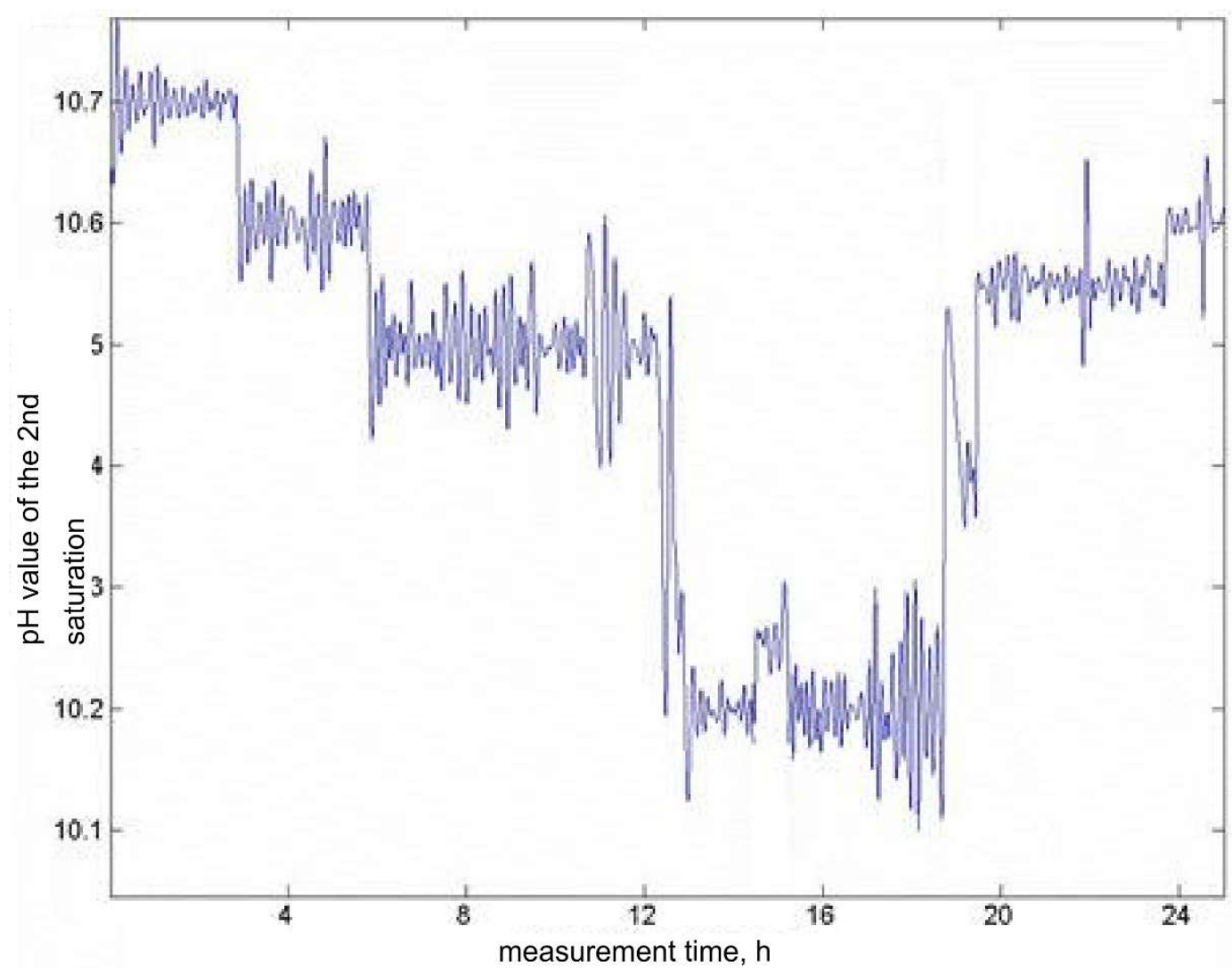

Fig. 4. Time series of $\mathrm{pH}$ value of the second saturation.

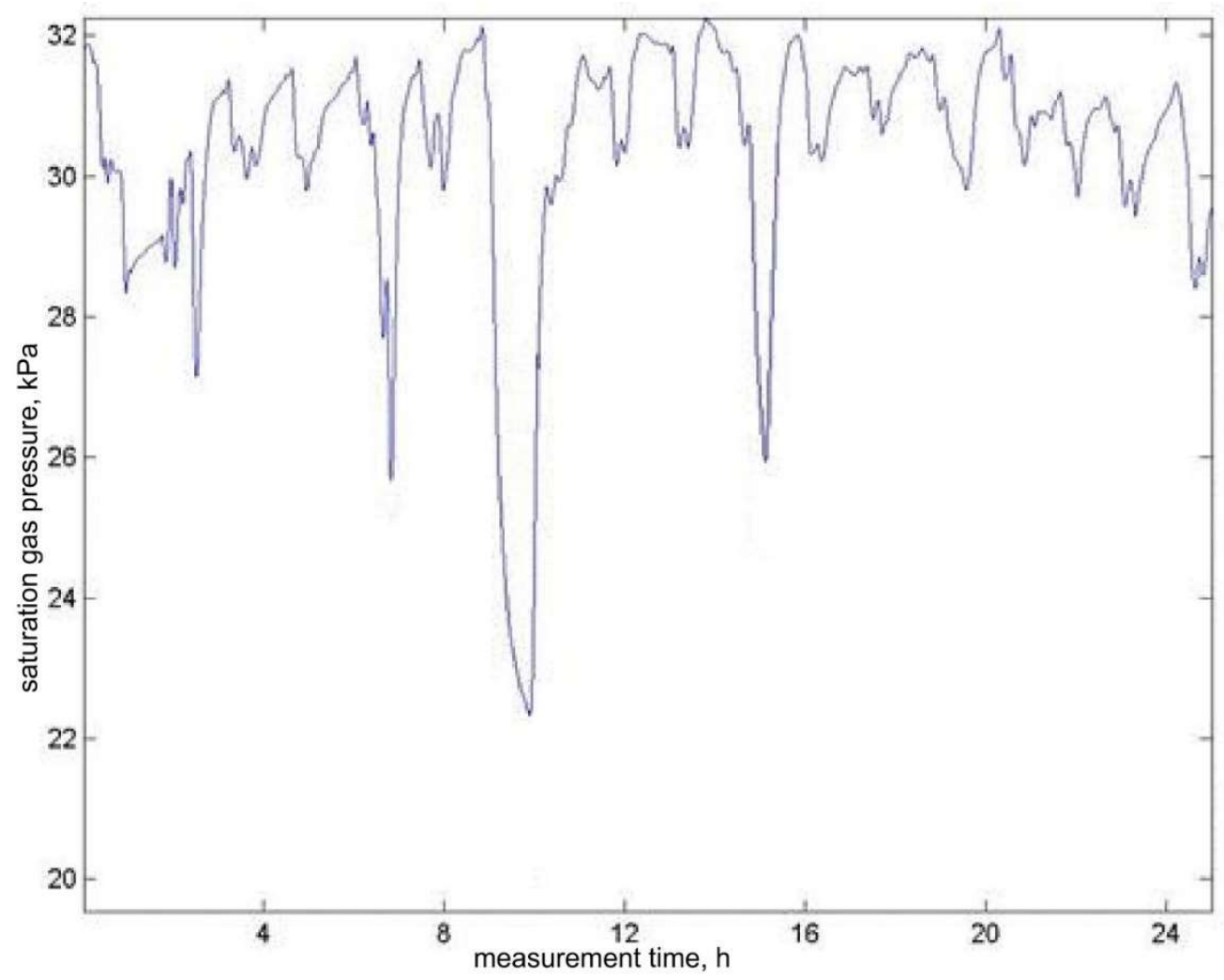

Fig. 5. Time series of $\mathrm{CO} 2$ saturation gas pressure. 
Today, for the study of dynamical systems, it is important to calculate the indicators of the attractor of some dynamical system, the mathematical models of which are unknown, but, as a rule, the dimension of the phase space is also unknown. In this situation, we have information about the behavior over time of individual variables that characterize the object of control.

To calculate such parameters as dimension, entropy, spectrum of Lyapunov, Hirst and other characteristics of attractors, it is necessary to have a set of points defined in the phase space of dimension $n$ and, which belong to the attractor [320].

Let any process variable be determined by dynamic discrete equations in the state space:

$$
\begin{gathered}
x(t+1)=F(x(t)), \\
y=Q(x(t)),
\end{gathered}
$$

where $x(t)$ is an $n$-dimensional vector of system states; $y(t)$ - measured (initial) process; $t$ - discrete time, $F, Q$ - nonlinear operators.

If we linearize equation (13) within the trajectory of motion, we obtain the relation:

$$
\begin{array}{r}
x(t+1)=A x(t), \\
y(t)=C x(t) .
\end{array}
$$

Here $C$ is the dimension matrix $(1 \times n){ }^{A=\frac{d}{d x} F(x, t)}$ - Jacobi dimension matrix $(n$ $\times n$ ), which determines the stability properties of the trajectory.

It is necessary to build a phase trajectory of the system, to determine the nature of stability or instability.

The states to which the system aspires when changing external parameters are called points of attraction. The minimum set of points to which almost all trajectories 
aspire is called the attractor. Packard's method can be used to construct the attractor of the system according to experimental data [324].

According to Takens's theorem [320], there is a vector function $\Lambda$ that reflects the space of states of the system in the Euclidean space of dimension $m$ :

$$
\mathrm{z}_{\mathrm{i}}=\Lambda\left(\mathrm{x}_{\mathrm{i}}\right), \mathrm{z}_{\mathrm{i}} \in \mathrm{R}^{m},
$$

The characteristics of both systems are invariant, which allows us to determine them from experimental data without knowing all the variables of a dynamic system. The dimension $\mathrm{m}$ can be determined, for example, by the methods of symmetry theory [325].

To find the vectors $z_{i}$ of the space $R_{m}$ in the time series, Packard proposed to use vectors derived from elements of the series on the same principles as in the autoregression equations:

$$
z_{i}=\left\{x_{i}, x_{i+1}, \ldots, x_{i+m-1}\right\}^{T},
$$

or

$$
z_{i}=\{x(t), x(t+\tau), \ldots, x(t+(m-1) \tau)\}^{T},
$$

where $x_{i}$ is the $i$-th element of the time series, $m$ is the dimension of the embedding space.

The resulting trajectory should not contain self-intersections, however, selfintersections in the array of discrete points $z$ usually do not occur, so look for so-called "close neighbors" - pairs of vectors that were close in the reconstruction, but their prototypes were far away. In other words, let $z_{i}(m)$ and $z_{j}(m)$ be two close neighbors in the reconstruction of dimension $m$, and let $z_{i}(m+1)$ and $z_{j}(m+1)$ correspond to them in reconstruction of dimension $m+1$.

To determine the optimal value of the delay time ta method [326] based on information theory is used, which uses the first minimum of mutual information for $x_{i}$ and $x_{i+1}$. Histograms approximating the distributions $x_{i}$ and $x_{i+1}$ and compatible 
distributions $x_{i}$ and $x_{i+1}$ are constructed in time series. Entropy and mutual information are calculated on the basis of histograms:

$$
S=-\sum p_{i j}(\tau) \log _{2} \frac{p_{i j}(\tau)}{p_{i} p_{j}}
$$

where $p_{i}$ is the probability of finding a point in the $i$-th interval; $p_{i j}(\tau)-$ total probability hits $x_{i}$ in the $i$ - th interval and hits $x_{i+1}$ в $j$ - th interval.

To build an attractor, it is necessary to filter. The following method is used [327]. For each vector $z_{i}$ of the obtained reconstruction, the nearest neighbors are calculated such that $\left\|z_{i}-z_{j}\right\|<\underline{\varepsilon}$. The value of $\varepsilon$ is set based on a priori estimates of the amount of noise or by sequential approximation. For each vector $z_{i}=\left\{x_{i}, x_{i+1}, \ldots, x_{i+m}-\right.$ $1\}^{\mathrm{T}}$ the adjusted value of $z \cdot$ is calculated by averaging over all $z^{\prime}$ nearest neighbors:

$$
z^{\bullet}=\frac{1}{N} \sum_{k-1}^{N} z_{k}^{\prime}
$$

After full passage on all points of the reconstructed trajectory all points, except the first and last $(m-1) / 2$, points will be corrected.

The study of the main characteristics of time series is carried out in the software environment Fractan. The results of experimental studies for the defecosaturation system are presented below.

Based on the study of the time series of the $\mathrm{pH}$ value of the second saturation, the Hirst index is $H=0.7446$, with the fractal dimension $D=2-\mathrm{H}=1.2554$. The optimal delay $\tau=26$. The maximum dimension of the phase space is 3 .

Also, the Hearst index is calculated for the time series of saturation gas pressure, which is $H=0.7148$, with the fractal dimension $D=2-\mathrm{H}=1.2852$. The optimal delay $\tau=128$. The maximum dimension of the phase space is 4 . And for juice consumption $-\mathrm{H}=0.9846, \mathrm{D}=2-\mathrm{H}=0.1621, \tau=67$, the dimension of the phase space is 5 . 


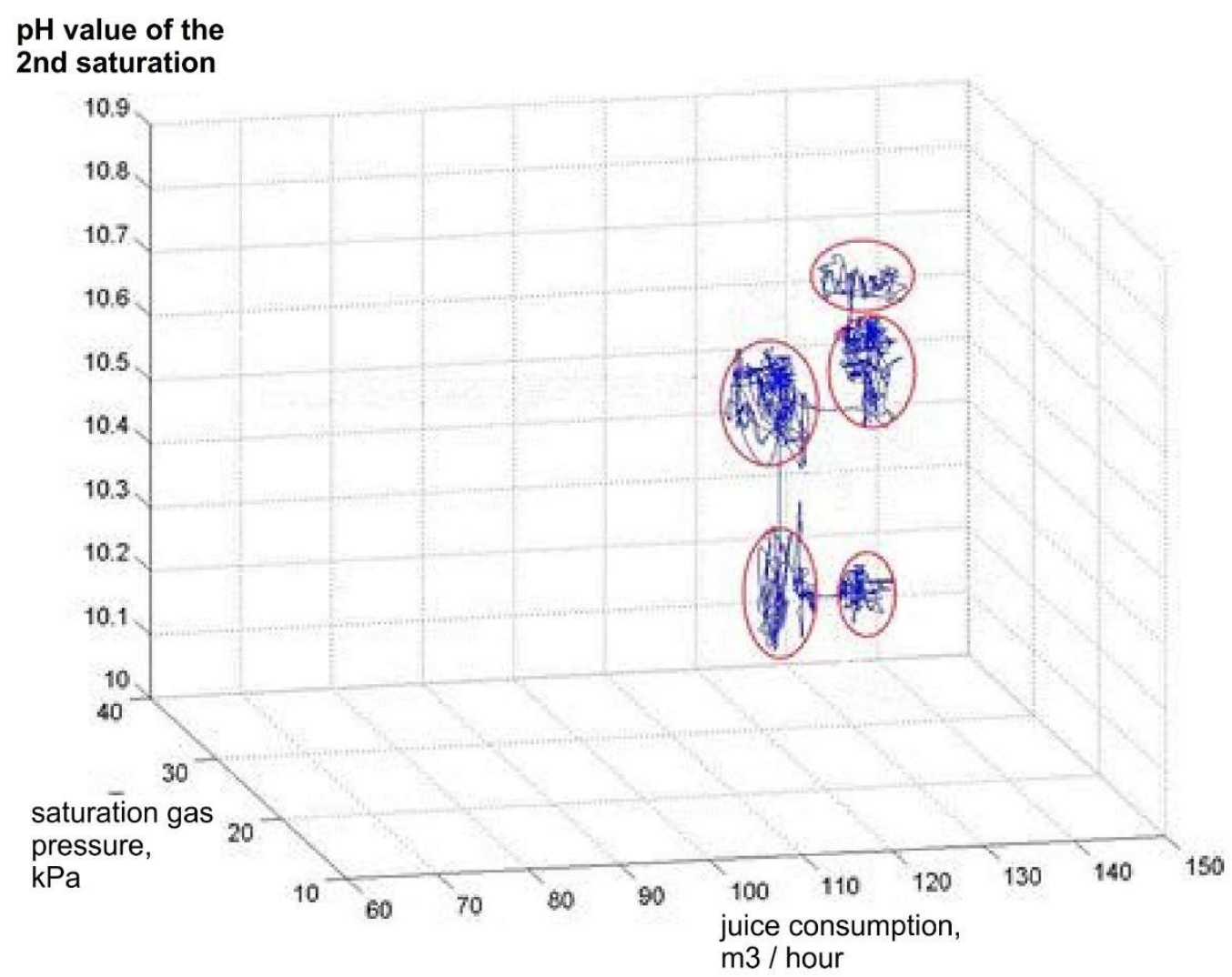

Fig.6. Reconstructed phase portrait with areas of attraction.

Estimating the Hirst index, we can say with all probability that these time series, and accordingly the control object on which they are obtained, is a complex nonlinear time system. Reconstruction of the attractor is carried out in the software environment MATLAB ${ }^{\circledR}$.

In the reconstructed phase space (Fig. 6) the traction regions, the attractors of the dynamic system, which are shifted to the planes of $\mathrm{pH}$ and saturation gas pressure, are clearly visible.

\section{Conclusions and results}

The attractive behavior of the control object, juicing department, from the standpoint of deterministic chaos is investigated.

Studies confirm the hypothesis of nonlinear complex behavior of a dynamic control object, the presence in its behavior of deterministic chaos, the phenomena of intermittency and attractive behavior.

The tasks of deeper research of the object of control and search of ways and possibilities of forecasting with the set depth of the forecast of behavior are set. 